\title{
O desmatamento na Amazônia e a importância das áreas protegidas
}

LEANDRO VALLE FERREIRA, EDUARDO VENTICINQUE e SAMUEL ALMEIDA

\section{Contextualização}

$\mathrm{O}$ BIOMA AMAZÔNIA estende-se do oceano Atlântico às encostas orientais da Cordilheira dos Andes, até aproximadamente $600 \mathrm{~m}$ de altitude, contendo parte de nove países da América do Sul, sendo 69\% dessa área pertencente ao Brasil (Ab'Saber, 1977). Esse bioma abrange os estados do Pará, Amazonas, Maranhão, Goiás, Mato Grosso, Acre, Amapá, Rondônia e Roraima, totalizando $4.871 .000 \mathrm{~km}^{2} \mathrm{e}$ uma população em torno de vinte milhões de habitantes, 60\% dela vivendo em áreas urbanas (Inpe, 2004).

O modelo tradicional da ocupação da Amazônia tem levado a um aumento significativo do desmatamento na Amazônia legal, sendo este um fenômeno de natureza bastante complexa, que não pode ser atribuído a um único fator (Alencar et al., 2004).

As questões mais urgentes em termos da conservação e uso dos recursos naturais da Amazônia dizem respeito à perda em grande escala de funções críticas da Amazônia frente ao avanço do desmatamento ligado às políticas de desenvolvimento na região, tais como especulação de terra ao longo das estradas, crescimento das cidades, aumento dramático da pecuária bovina, exploração madeireira e agricultura familiar (mais recentemente a agricultura mecanizada), principalmente ligada ao cultivo da soja e algodão (Fearnside, 2003, Alencar et al., 2004 e Laurance et al., 2004).

Esse aumento das atividades econômicas em larga escala sobre os recursos da Amazônia legal brasileira tem aumentado drasticamente a taxa de desmatamento que, no período de 2002 e 2003 , foi de $23.750 \mathrm{~km}^{2}$, a segunda maior taxa já registrada nessa região, superada somente pela marca histórica de $29.059 \mathrm{~km}^{2}$ desmatados em 1995 (Inpe, 2004).

A situação é tão crítica que, recentemente, o governo brasileiro criou um Grupo Interministerial a fim de combater o desmatamento e apontar soluções de como minimizar seus efeitos na Amazônia legal (MMA, 2004).

Este trabalho pretende, portanto, mostrar como está distribuído espacialmente o desmatamento na Amazônia legal, quais são suas conseqüências ambientais além de apontar algumas propostas de soluções para a diminuição do desmatamento. 


\section{Resultados}

O processo de desmatamento normalmente começa com a abertura oficial ou clandestina de estradas que permitem a expansão humana e a ocupação irregular de terras à exploração predatória de madeiras nobres. Posteriormente, converte-se a floresta explorada em agricultura familiar e pastagens para a criação extensiva de gado, especialmente em grandes propriedades, sendo este fator responsável por cerca de $80 \%$ das florestas desmatadas na Amazônia legal. Mais recentemente, as pastagens estão dando lugar à agricultura mecanizada, principalmente àquela ligada às culturas de soja e algodão (Figura 1 ).

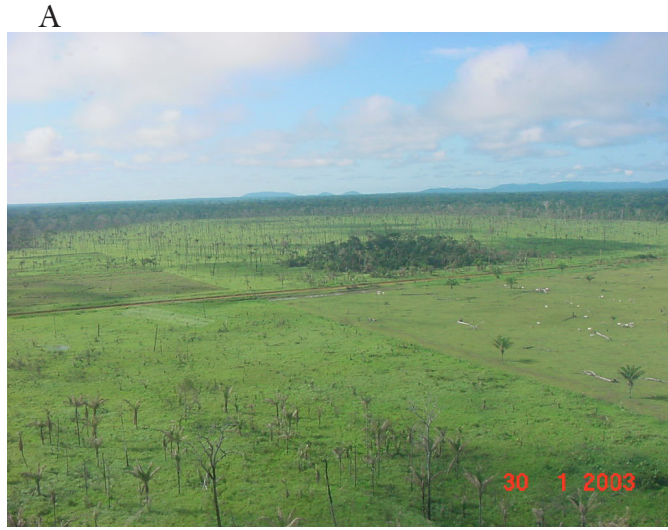

C

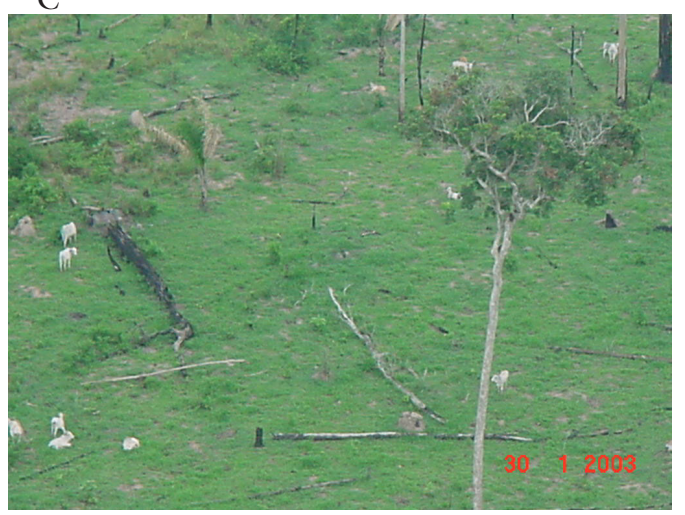

B

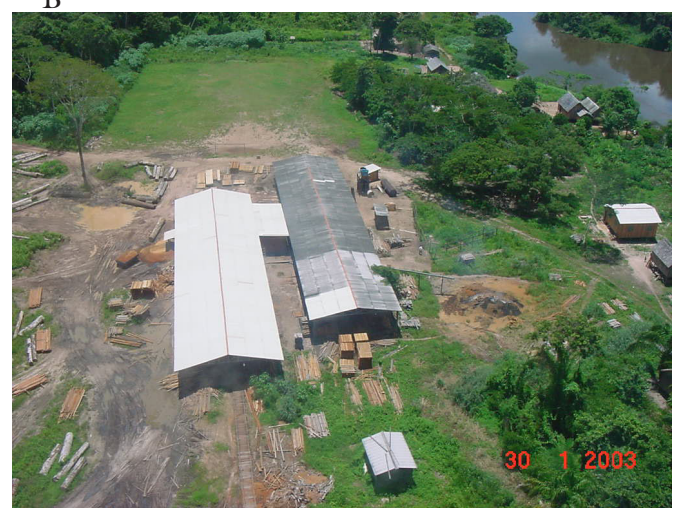

$\mathrm{D}$

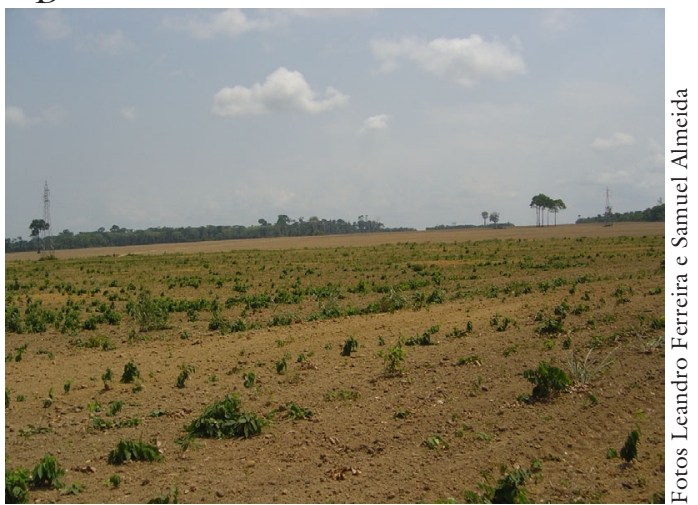

Figura l - Etapas do modelo tradicional de ocupação da Amazônia legal:

(A) abertura de estradas, (B) extração seletiva de madeiras nobres,

(C) pecuária extensiva e (D) agricultura tradicional ou mecanizada.

Como conseqüência dessas atividades, o desmatamento na Amazônia legal brasileira tem apresentado uma relação crescente nos últimos dez anos, com um aumento significativo entre os anos de 2001-2002 e 2002-2003 (Figura 2).

Existe uma relação direta entre a economia, o avanço da fronteira na Amazônia legal e a taxa de desmatamento crescente desde 1990, influenciada pelo estado da economia nacional. Contudo, nos últimos anos, essa relação começou a modificar-se, pois a taxa de desmatamento foi crescente, apesar da falta de crescimento econômico (Figura 2). 


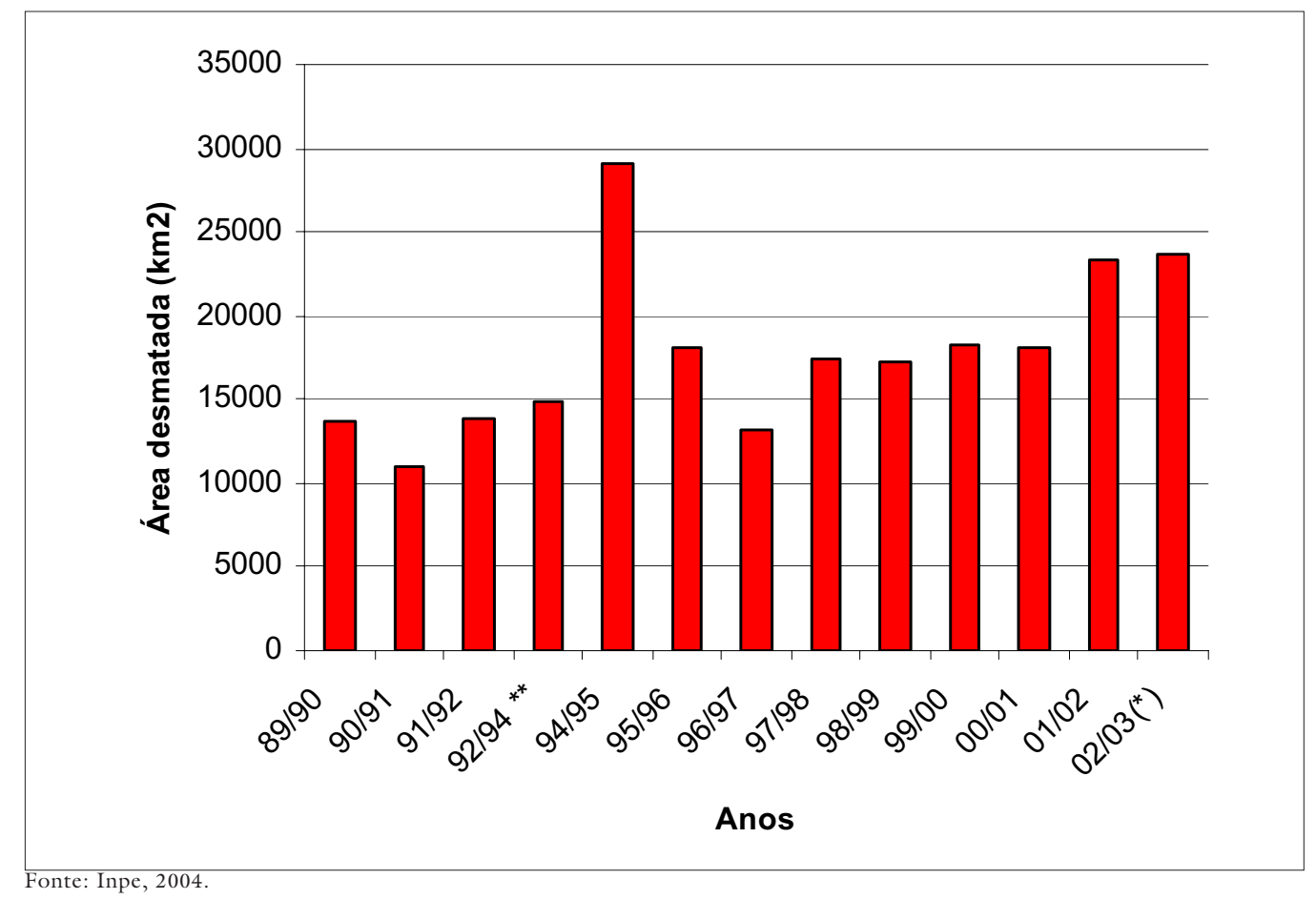

Figura 2 - Total de área desmatada na Amazônia legal entre 1989-2003.

Isso sugere que uma nova dinâmica está influenciando o desmatamento nessa região, tais como uma dinâmica ligada ao mercado de exportação impulsionada pela alta rentabilidade das principais atividades econômicas, como a extração madeireira à pecuária e, mais recentemente, a agroindústria (Fearnside, 2003 e Alencar et al., 2004)

A área cumulativa desmatada na Amazônia legal brasileira chegou a cerca de 653 mil km², em 2003, correspondendo a 16,3\%. Contudo, esse desmatamento não é distribuído homogeneamente, mas sim concentrado ao longo do denominado "arco do desmatamento", cujos limites se estendem do sudeste do estado do Maranhão, ao norte do Tocantins, sul do Pará, norte de Mato Grosso, Rondônia, sul do Amazonas e sudeste do estado do Acre (Figura 3).

Os estados que mais desmataram a Amazônia brasileira entre 2001 e 2003 foram os do Pará, Rondônia, Mato Grosso e Maranhão, que, juntos, corresponderam por mais de $90 \%$ do desmatamento observado nesse período (Tabela 1 ).

\section{As estradas e o desmatamento na Amazônia}

A proporção do desmatamento como função da distância das estradas na Amazônia legal tem, normalmente, padrões exponenciais, ou seja, grande proporção de desmatamento próximo às estradas (Figura 4).

Esses padrões já foram observados por diversos estudos realizados na nessa região. Ferreira (2001) encontrou curvas exponenciais do desmatamento em função da distância das estradas. Nepstad et al. (2001) demonstraram que três 
quartos dos desmatamentos entre 1978 e 1994 ocorreram dentro de uma faixa de $100 \mathrm{~km}$ de largura ao longo das rodovias BR 010 (Belém-Brasília), BR 364 (Cuiabá-Porto Velho) e PA 150.

Esses resultados são extremamente importantes, em termos de políticas públicas para a Amazônia, pois são nessas áreas, sob o auspício do Programa Avança Brasil, que o governo pretendia investir cerca de quarenta bilhões de dólares na construção e recuperação de cerca de $7.500 \mathrm{~km}$ de estradas, aumentando drasticamente a acessibilidade a muitas áreas remotas (Laurance et al., 2004).

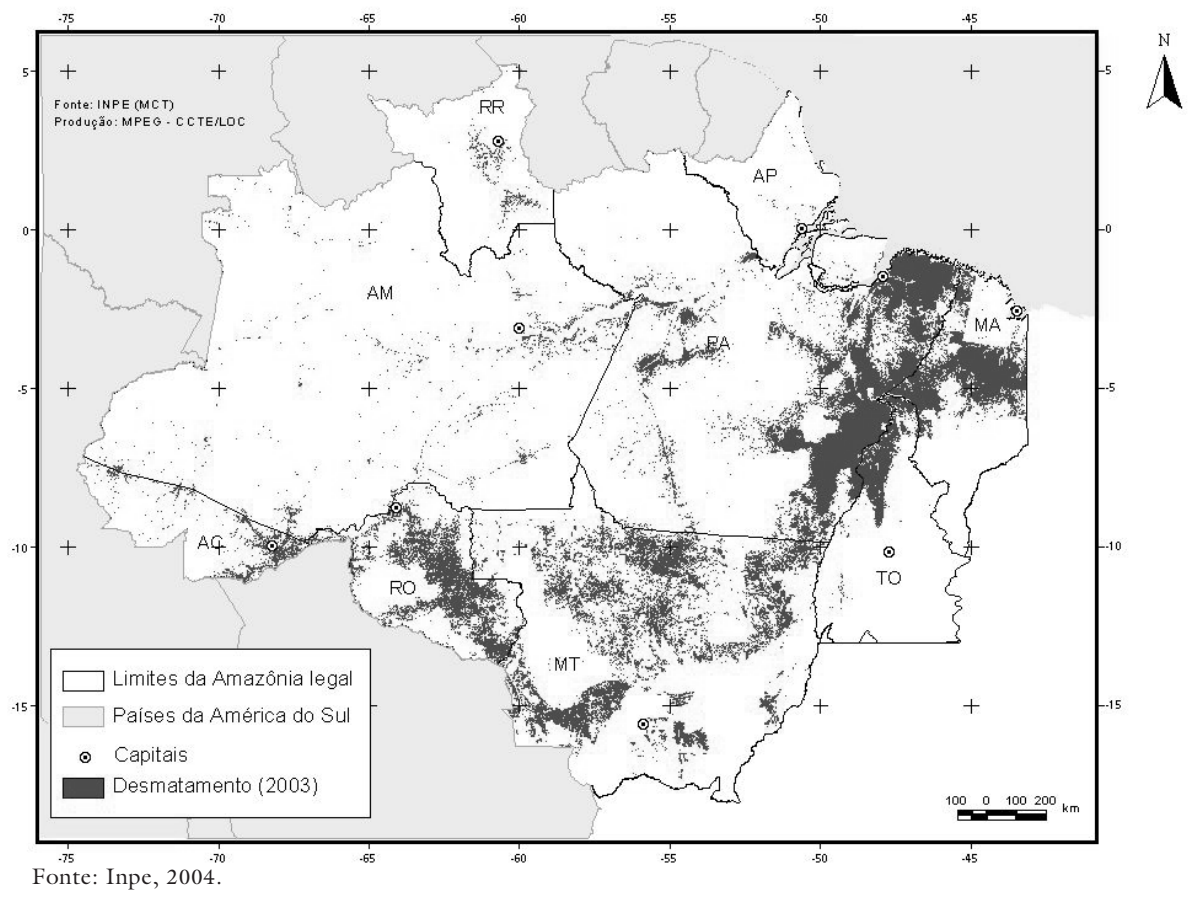

Figura 3 - Área total desmatada na Amazônia legal.

Anos

\begin{tabular}{c|c|c|c|c|c|c}
\hline & 2001 & & 2002 & & 2003 & \\
\hline Estados & $\mathrm{Km}^{2}$ & $\%$ & $\mathrm{Km}^{2}$ & $\%$ & $\mathrm{Km}^{2}$ & $\%$ \\
\hline $\mathrm{MT}$ & 7.703 & 42 & 7.578 & 33 & 10.416 & 44 \\
\hline $\mathrm{PA}$ & 5.237 & 29 & 8.697 & 37 & 7.293 & 31 \\
\hline $\mathrm{RO}$ & 2.673 & 15 & 3.605 & 15 & 3.463 & 15 \\
\hline $\mathrm{MA}$ & 958 & 5 & 1.330 & 6 & 766 & 3 \\
\hline Total & $\mathbf{1 6 . 5 7 1}$ & $\mathbf{9 1}$ & $\mathbf{2 1 . 2 1 0}$ & $\mathbf{9 1}$ & $\mathbf{2 1 . 9 3 8}$ & $\mathbf{9 2}$ \\
\hline $\begin{array}{c}\text { Outros } \\
\text { Estados }\end{array}$ & 1594 & 9 & 2.056 & 9 & 1812 & 8 \\
\hline $\begin{array}{c}\text { Amazônia } \\
\text { Legal }\end{array}$ & 18.165 & & 23.266 & & 23.750 & \\
\hline
\end{tabular}

Tabela 1 - Área e proporção total de desmatamento observado nos estados da Amazônia legal (Inpe, 2003). 


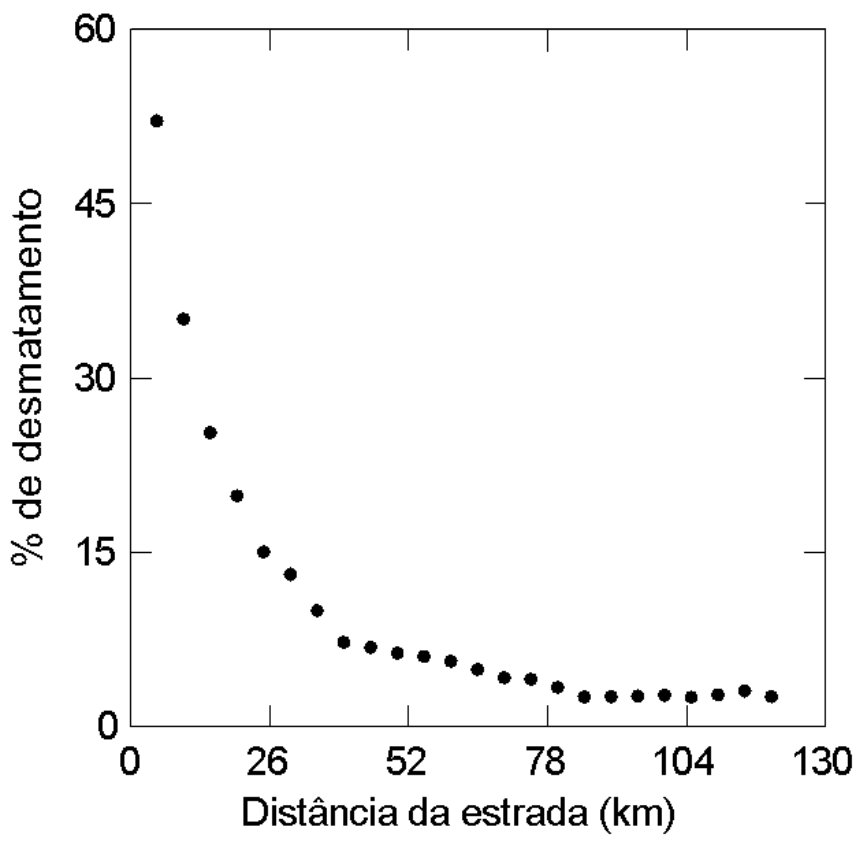

Figura 4 - Proporção de desmatamento em função da distância da estrada na Amazônia legal (Ferreira, 2001).

\section{As áreas protegidas e o desmatamento na Amazônia legal}

O Brasil possui diversas categorias de unidades de conservação, nos níveis federal, estadual e municipal. Conforme o Sistema Nacional de Unidades de Conservação da Natureza (SNUC), Lei Federal nº 9.985/00 (Ibama, 2004).

As unidades de uso sustentável visam a compatibilizar a conservação da natureza com o uso sustentável de seus recursos naturais, ou seja, permite-se a exploração e o aproveitamento econômico direto de forma planejada e regulamentada. Nesse grupo estão incluídas as categorias de manejo Área de Proteção Ambiental, Área de Relevante Interesse Ecológico, Floresta Nacional, Reserva Extrativista, Reserva de Fauna e Reserva de Desenvolvimento Sustentável (Ibama, 2004).

O grupo das unidades de proteção integral visa à conservação da biodiversidade, e inclui as categorias Estação Ecológica, Reserva Biológica, Parque Nacional, Monumento Natural e Refúgio de Vida Silvestre. Essas categorias de manejo destinam-se à preservação integral da biota e demais atributos naturais existentes em seus limites, conciliados à realização de pesquisas científicas, as quais devem ser autorizadas pelo Ibama, estando sujeitas'às normas por este estabelecida. Algumas delas admitem a visitação pública para objetivos educacionais amplos ou restritos, mas estão sempre sujeitas às normas estabelecidas pelo seu órgão responsável e por aquelas previstas em regulamento (Ibama, 2004).

Outras categorias de áreas institucionais na Amazônia legal são as Terras Indígenas, sob jurisdição do governo federal, por meio da Fundação Nacional 
do Índio (Funai). Diversos autores têm discutido se essas áreas podem ser consideradas "áreas protegidas", já que as mesmas não obedecem aos critérios estabelecidos pelo SNUC. Contudo, essa discussão não é o objetivo deste texto. Neste estudo, as Unidades de Conservação de Proteção Integral, Uso Sustentável e as Terras Indígenas na Amazônia legal serão denominadas de "áreas protegidas".

Além de determinarmos como está distribuído espacialmente o desmatamento na Amazônia legal brasileira, uma questão importante é saber como está distribuída a proporção desse desmatamento dentro e fora do atual conjunto das Unidades de Conservação e Terras Indígenas na Amazônia. Em outras palavras, esse conjunto de áreas protegidas pode ser um instrumento de política pública usado para a diminuição do desmatamento na Amazônia? Ou devido à sua baixa implementação esse conjunto não cumpre sua função de diminuir o avanço do desmatamento na Amazônia legal brasileira? (Aaron et al., 2001).

As Unidades de Conservação de Proteção Integral e Uso Sustentável (Estadual e Federal), Terras Indígenas e o desmatamento na Amazônia legal ocupam atualmente $4,9 \%, 9,1 \%, 20,4 \%$ e 16,5\%, respectivamente (Figura 5).

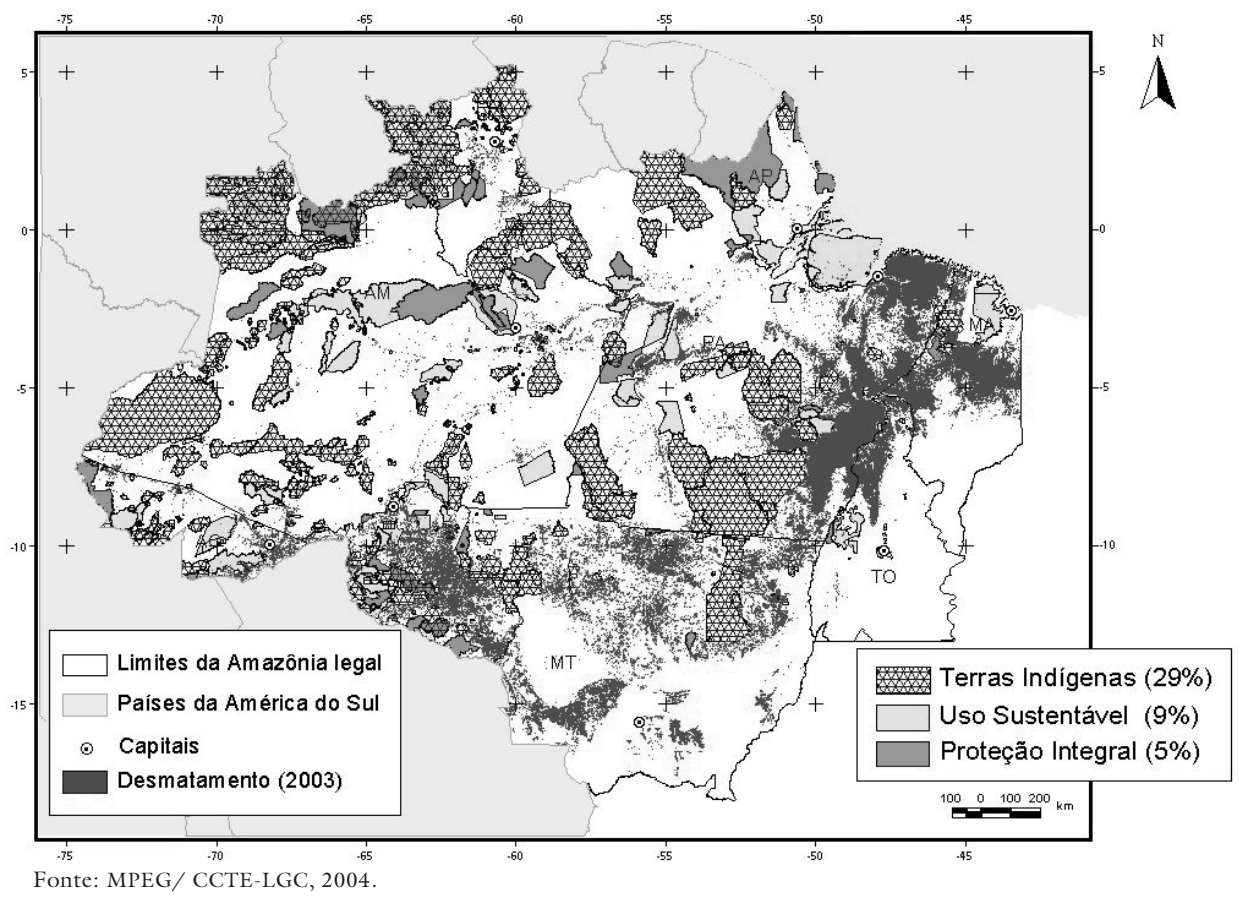

Figura 5 - Proporção de área ocupada das Unidades de Conservação e Terras Indígenas na Amazônia legal e o desmatamento observado.

Atualmente, a proporção total de desmatamento nos estados do Mato Grosso, Pará e Rondônia é de $28,4 \%, 20,4 \%$ e $29,2 \%$, respectivamente. Contudo, existe uma grande diferença na proporção desse desmatamento dentro ou fora das áreas protegidas nesses estados (Figura 6). 


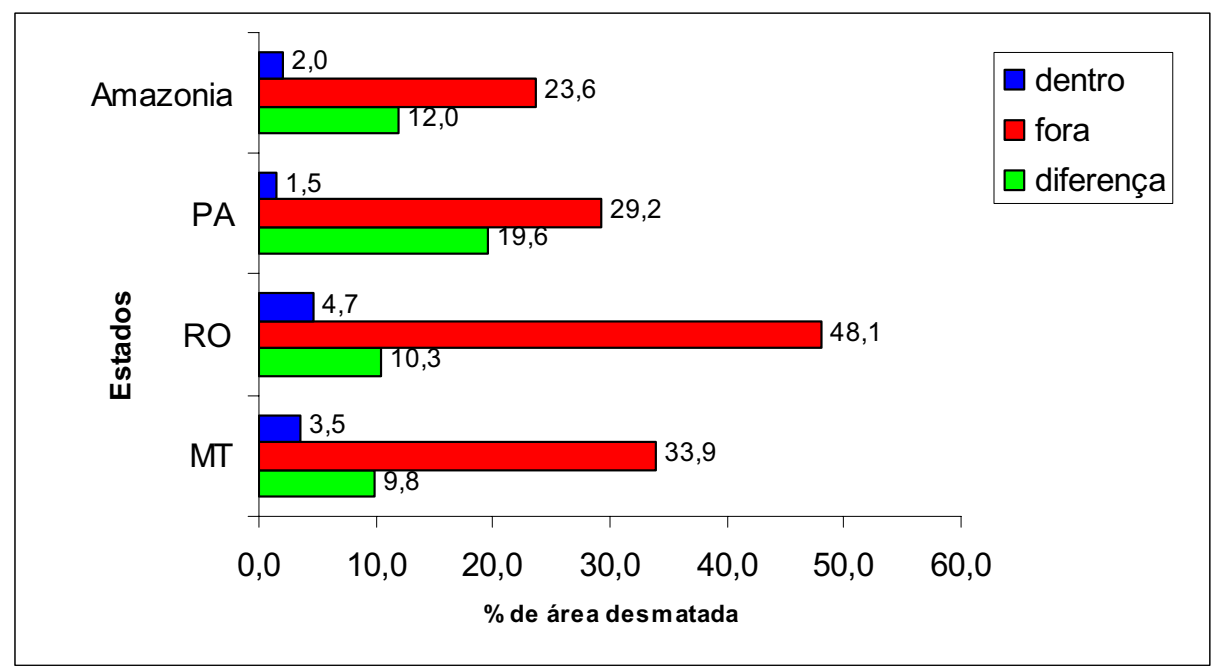

Figura 6 - Proporção do desmatamento dentro e fora das áreas protegidas na Amazônia legal e nos estados de Mato Grosso (MT), Pará (PA) e Rondônia (RO).

A proporção de área desmatada dentro das áreas protegidas variou de 1,5 a $4,7 \%$, enquanto a proporção de desmatamento fora delas variou de $29,2 \%$ a $48,1 \%$ nos três estados analisados (Figura 6).

A diferença do desmatamento dentro ou fora das áreas protegidas variou de aproximadamente dez vezes nos estados de Mato Grosso e Rondônia a aproximadamente vinte vezes no estado do Pará (Figura 6).

Esses resultados demonstram claramente a importância das áreas protegidas (Unidades de Conservação e Terras Indígenas) como uma das ferramentas para conter ou diminuir o processo do desmatamento nos três estados que mais contribuíram com o desmatamento na Amazônia legal e contraria parcialmente a hipótese generalizada de que as áreas protegidas na Amazônia não estão cumprindo sua função principal na conservação e uso racional dos recursos na região, pelo fato de que muitas não estão ainda implementadas e apresentam diferentes graus de vulnerabilidade (Sá e Ferreira, 2000).

\section{Ordenamento territorial na Amazônia}

Recentemente, a revista The Economist publicou uma reportagem sobre as conseqüências socioambientais do asfaltamento da rodovia Cuiabá-Santarém (BR163), já que as estradas são reconhecidamente um dos maiores responsáveis pelo desmatamento na Amazônia legal. Uma das conclusões da reportagem foi uma indagação: “É possível conciliar o desenvolvimento e a conservação da Amazônia?”.

A resposta para essa questão não é simples, mas existe essa possibilidade, e esta é possível através do processo de ordenamento territorial da Amazônia legal, usando como instrumento de aplicação o zoneamento ecológico-econômico.

De maneira simplificada, a ocupação ordenada na Amazônia de forma a reduzir a conversão de ambientes pelo desmatamento pode ser resumida na se- 
guinte a fórmula: "10-20-30-40” que conciliariam as questões de preservação e uso dos recursos da Amazônia legal brasileira (Tabela 2).

\begin{tabular}{l|c|c|l}
\hline Condição & $\begin{array}{c}\text { Situação } \\
\text { Atual }\end{array}$ & $\begin{array}{c}\text { Situação } \\
\text { Pretendida }\end{array}$ & Como Fazer \\
\hline $\begin{array}{l}\text { Preservação } \\
\text { Ambiental }\end{array}$ & $5 \%$ & $10 \%$ & $\begin{array}{l}\text { Ampliação do atual sistema de } \\
\text { Unidades de Proteção Integral. }\end{array}$ \\
\hline $\begin{array}{l}\text { Uso econômico } \\
\text { tradicional }\end{array}$ & $17 \%$ & $20 \%$ & $\begin{array}{l}\text { Incentivo a atividade de pecuária, } \\
\text { agricultura, mineração e expansão } \\
\text { urbana e rural. }\end{array}$ \\
\hline Terras Indígenas & $29 \%$ & $30 \%$ & $\begin{array}{l}\text { Demarcação efetiva das Terras } \\
\text { Indígenas. }\end{array}$ \\
\hline Uso econômico & $9 \%$ & $40 \%$ & $\begin{array}{l}\text { Manutenção de áreas de floresta, } \\
\text { evitando o corte raso, através do } \\
\text { uso terras públicas (Unidades de } \\
\text { Conservação de Uso Sustentável) } \\
\text { e terras privadas, respeitando } \\
\text { a Reserva Legal e Áreas } \\
\text { de Preservação Permanente }\end{array}$ \\
\hline
\end{tabular}

Tabela 2 - Ordenamento territorial proposto para a Amazônia legal.

Essa proposta de ordenamento territorial contempla todas as atividades possíveis de serem realizadas na Amazônia legal. Existe, por exemplo, espaço para o aumento da expansão das atividades econômicas tradicionais, como pecuária e agricultura, que atualmente já desmataram cerca de $17 \%$ da região, podendo esta expansão chegar a $20 \%$. Contudo, outras ações devem ser implementadas, como o aumento da preservação em Unidades de Conservação de Proteção Integral; o respeito às Terras Indígenas e principalmente o uso econômico florestal da Amazônia, em terras públicas e privadas (Tabela 2).

É preciso romper com o modelo de ocupação tradicional da Amazônia, não se pode encarar a Amazônia legal como uma região a ser explorada irracionalmente, o ordenamento Territorial através do Zoneamento Ecológico-Econômico (ZEE) pode ser uma solução para subsidiar as decisões de planejamento socioambiental e desenvolvimento econômico em bases sustentáveis.

Bibliografia

AARON, G. B; RAYMOND, E. G.; RICE, R. E. e FONSECA, G. A. "Effectiveness of Parks in Protecting Tropical Biodiversity". Science 291, 2001, pp. 125-128.

ALENCAR, A.; NEPSTAD, N; MCGRATH, D; MOUTINHO, P; PACHECO, P; DIAZ, M. D. C. V e FILHO, B. S. Desmatamento na Amazônia: indo além da emergência crônica. Manaus, Instituto de Pesquisa Ambiental da Amazônia (Ipam), 2004, 89 p. 
FEARNSIDE, P. M. A floresta Amazônia nas mudanças globais. Manaus, Instituto Nacional de Pesquisas da Amazônia (Inpa), 2003, 134 p.

FERREIRA, L. V. “Identificação de áreas prioritárias para a conservação da biodiversidade por meio da representatividade das unidades de conservação e tipos de vegetação nas ecorregiões da Amazônia brasileira”, em CAPOBIANCO, J. P. R. (ed.). Biodiversidade na Amazônia brasileira: avaliação e ações prioritárias para a conservação, uso sustentável e repartição de benefícios. São Paulo, Instituto Socioambiental, 2001, pp. 268-286.

INSTITUTO NACIONAL DE PESQUISAS ESPACIAIS (Inpe). Monitoramento da Floresta, São José dos Campos 2004.

AMAZÔNICA BRASILEIRA POR SATÉLITE PROJETO PRODES (www.dpi.inpe.br/ prodesdigital).

LAURANCE, W. F.; COCHRANE, M. A.; BERGEN, S.; FEARNSIDE, P. M.; DELAMÔNICA, P.; BARBER, C.; D’ANGELO, S. e FERNANDES, T. “The Future of the Brazilian Amazon". Science 291, 2001, pp. 438-439.

LAURANCE, W. L.; ALBERNAZ. A. K. M.; FEARNSIDE, P. M.; VASCONCELOS, H; FERREIRA, L. V. "Deforestation in Amazonia". Science 304, 2004, pp. 1109 1111 .

MINISTÉRIO DO MEIO AMBIENTE (MMA). Plano de ação para a prevenção e controle do desmatamento na Amazônia legal, Brasília, 2004.

NEPSTAD, D.; CARVALHO, G.; BARROS, A. C.; ALENCAR, A; CAPOBIANCO, J. B.; BISHOP, J; MOUTINHO, P.; LEFEBVRE, P. e SILVA, U. L. “Road Paving, Fire Regime Feedbacks, and the Future of Amazon Forests". Forest Ecology and Management 5524, 2001, pp. 1-13.

SÁ, R. M. L e FERREIRA, L. V. Áreas protegidas ou espaços ameaçados: o grau de implementação e vulnerabilidade das unidades de conservação federal brasileiras de uso indireto. Brasília, Série Técnica III, WWF Brasil, 2000.

RESUMO - O MODELO da ocupação demográfica da Amazônia legalnos últimos cinqueta anos tem levado a níveis significativos de desmatamento, resultante de múltiplos fatores, tais como a abertura de estradas pioneiras, o crescimento das cidades, a ampliação de pecuária extensiva, a acelerada exploração madeireira e a crescente agricultura intensiva de mono-culturas. A área cumulativa desmatada na Amazônia legal brasileira chegou a cerca de $653.000 \mathrm{~km}^{2}$ em 2003, correspondendo a 16,3\% da região. Este estudo visou a deter-minar o desmatamento dentro e fora dos atuais Unidades de Conservação (UC) e Terras Indígenas (TI) na Amazônia legal, nos estados de Mato Grosso, Rondônia e Pará, que, juntos, corresponderam por mais de $90 \%$ do desmatamento observado entre 2001 e 2003. Os resultados mostraram que o desmatamento foi cerca de dez a vinte vezes menor dentro das Unidades de Conservação e Terras Indígenas do que em áreas contí-guas fora delas. Isto demonstra a importância dessas áreas protegidas para diminuir o processo do desmatamento nos três estados. Isto refuta a hipótese generalizada de que estas áreas não cumprissem a sua função principal na conservação e uso racional dos recursos na Amazônia legal. 
ABSTRACT - DEMOGRAPHIC occupation of the Brazilian Legal Amazon within the past 50 years has lead to significant levels of deforestation as the result of several factors such as new road construction, urban growth, expansion of extensive cattle ranching, increasing timber extraction, and rising intensive agriculture of monocultures. The cumulative deforested area in Brazil's Legal Amazonia was approximately $653,000 \mathrm{~km}^{2}$ in 2003 , correspondingto $16,3 \%$ of the region. This study sought to assess deforestation within or near to protected areas (conservation units) and indigenous reserves in Legal Ama-zonia in the states of Mato Grosso, Rondônia, and Pará that jointly were responsible for more than $90 \%$ of regional deforestation from 2001 to 2003 . Results show that deforestation was about 10 to 20 times less within the protected areas and indigenous lands than in adjoining areas. This demonstrates the importance of protected areas to hold back deforestation in these three states, refuting the widely held hypothesis that protected areas do not fulfill their main function in the conservation and sustainable use of resources in Amazonia.

Palavras-chave: Amazônia, Desmatamento, Áreas protegidas.

Key words: Amazon, Deforestation, Protected Areas.

Leandro Valle Ferreira é doutor em Biologia e trabalha no Museu Paraense Emílio Goeldi (MPEG).

Eduardo Venticinque é doutor em Ecologia, Wildlife Consevation Society (WCS) e email: eventicinque@wcs.org.

Samuel Almeida é mestre em Ecologia e trabalha no Museu Paraense Emílio Goeldi (MPEG).

Os autores destacam que este texto não teria sido concluído sem a ajuda do Programa Institucional de Biodiversidade da Amazônia do Museu Paraense Emílio Goeldi e das inúmeras sugestões dos membros da Confraria Cachorrada, Dário Amaral, Eduardo Lauande, Rafael Salomão, Samuel Almeida e Ulisses Galatti.

Texto recebido em $1^{\circ}$ de fevereiro de 2005 e aceito para publicação em 26 de fevereiro de 2005. 